\title{
Atemwegsmanagement in der Thoraxanästhesie mit dem Doppellumentubus
}

Torsten Loop, Johannes Spaeth
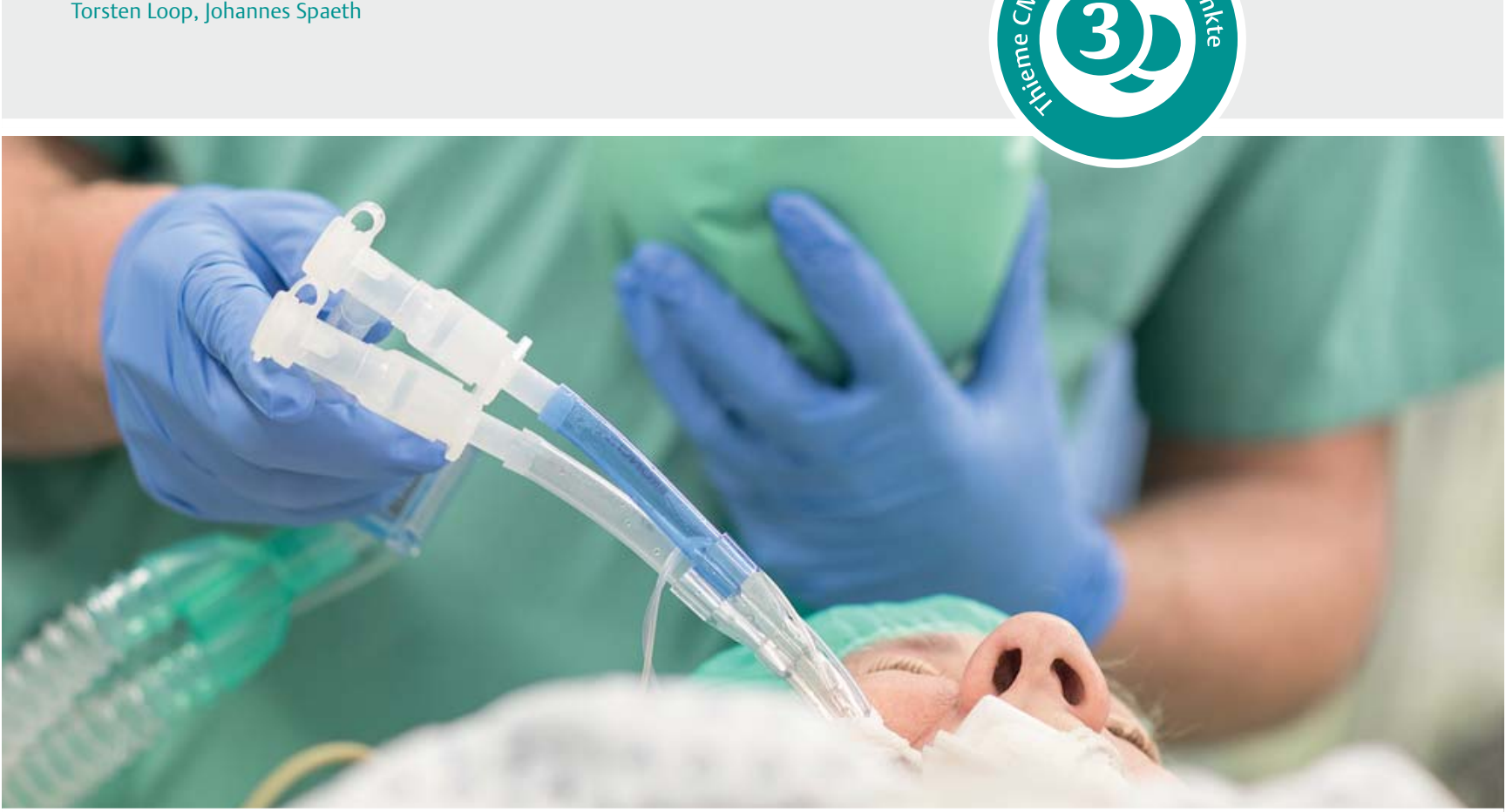

Quelle: KH Krauskopf.

Der Doppellumentubus (DLT) stellt nicht nur bei thoraxchirurgischen Eingriffen die am häufigsten angewandte Technik und Atemwegshilfe für die Seitentrennung der Lungen mit konsekutiver Ein-Lungen-Ventilation (ELV) dar. Dieser Artikel liefert grundlegende Empfehlungen und illustrative Darstellungen zum praktischen Management im klinischen Alltag.

\section{Anatomie}

Die Kenntnisse der tracheobronchialen bronchoskopischen Anatomie sind für das Atemwegsmanagement bei thoraxchirurgischen Eingriffen unabdingbar. Die Trachea weist distal der Stimmbandebene 16-20 Knorpelspangen auf, die etwa zwei Drittel der Zirkumferenz ausmachen. Die posteriore Wand ist knorpelfrei, fibroelastisch (Pars membranacea) und erlaubt durch den Längscharakter der Fasern eine gute fiberoptische Orientierung.

Die Trachea ist beim Erwachsenen 11-14 cm lang ( $<12 \mathrm{~cm}$ bei Frauen, bis $14 \mathrm{~cm}$ bei Männern). Der Abstand von den Lippen zur Hauptkarina beträgt zwischen $21 \mathrm{~cm}$ (Frauen) und $24 \mathrm{~cm}$ (Männer) und unterliegt anatomischen Varianten [1]. Der Tracheadurchmesser beträgt normalerweise geschlechtsabhängig 1,3-2,7 cm. Das Lumen des rechten Hauptbronchus ist weiter als das des linken. Dabei ist der rechte Hauptbronchus deutlich kürzer als der linke, da der Abgang des rechten Oberlappens schon nach etwa $2 \mathrm{~cm}$ erfolgt. Der tracheobronchiale Winkel ist mit $25-30^{\circ}$ rechts und $45^{\circ}$ links asymmetrisch [1]. Variationen in der tracheobronchialen Anatomie sind mit $10 \%$ häufig. Sie umfassen z. B. die Position des rechten Oberlappenbronchus, den Durchmesser von Trachea und Bronchien und pathologische Veränderungen [2-4].

Merke

Als Anästhesist sollte man sich der Variationen der tracheobronchialen Anatomie bewusst sein, um eine für den Patienten sichere Seitentrennung der Lungen zu gewährleisten. 


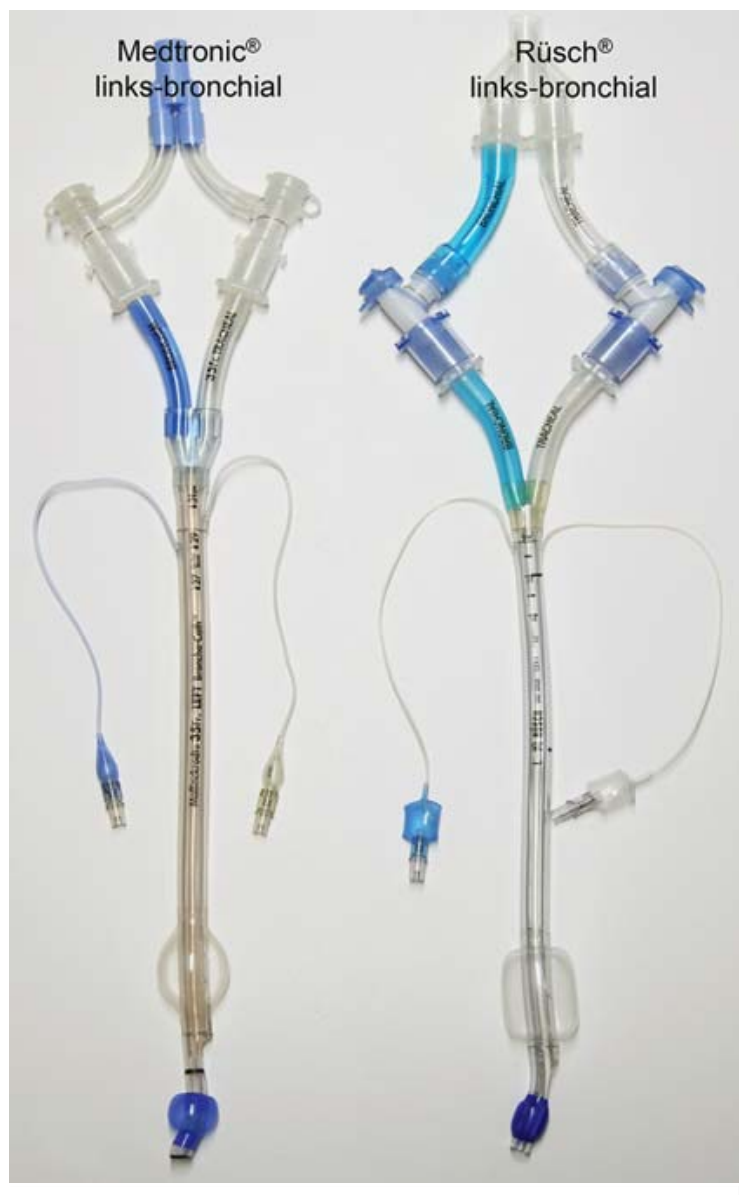

- Abb. 1 Exemplarische Darstellung zweier links-bronchialer Doppellumentuben. Unterschiede sind insbesondere im Cuff-Design, in der farblichen Kodierung und dem proximalen Ende (Bronchoskopie-Adapter, Verbindung zum Respirator) zu erkennen.

\section{Doppellumentubus}

Der Doppellumentubus ist ein ovaler, gabelförmiger Tubus mit einem trachealen und einem bronchialen Lumen und heutzutage als Einmalartikel mit Polyurethan-Cuff im Robertshaw-Design im Einsatz [5]. Das tracheale Lumen endet distal des trachealen Cuffs, das bronchiale Lumen am distalen Ende des Tubus ( $\bullet$ Abb. 1). Er wird kommerziell als linker und rechter DLT in den Größen $26 \mathrm{Fr}, 28 \mathrm{Fr}$, $32 \mathrm{Fr}, 35 \mathrm{Fr}, 37 \mathrm{Fr}$, $39 \mathrm{Fr}$ und $41 \mathrm{Fr}$ von verschiedenen Firmen angeboten ( $\bullet$ Abb. 1 u. 2, s. auch $\triangleright$ Tab. 2).

Der rechte DLT erlaubt über eine zusätzliche Öffnung oder einen Port die Ventilation des rechten Oberlappens ( Abb. 2). Designdetails wie der endobronchiale Winkel, Art des Cuffs, Lumendurchmesser und -lokalisation sind herstellerabhängig ( $\triangleright$ Abb. 1, 2 und $\mathbf{3}$ ). Generell sind sowohl der endobronchiale Cuff als auch das jeweilige proximale Ende blau eingefärbt ( $\triangleright$ Abb. 1 und 2). Mit dem VivaSight-DL ${ }^{\mathrm{TM}}$ existiert zusätzlich ein Doppellumentubus

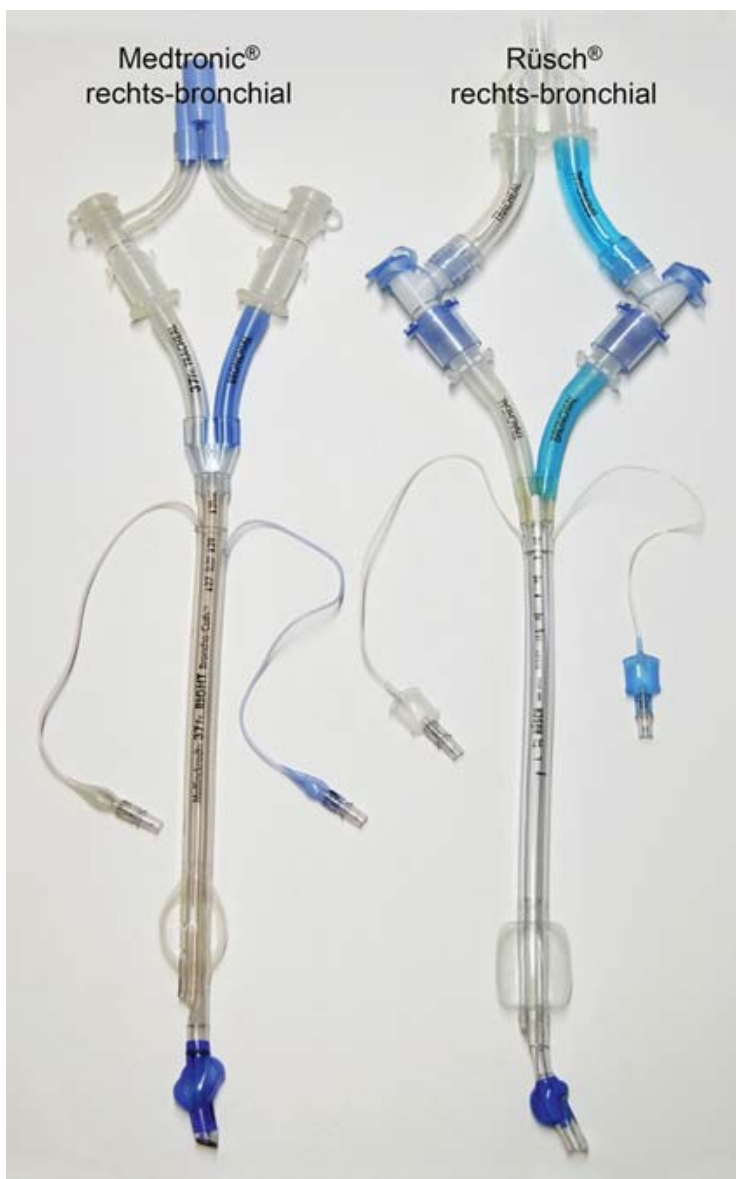

- Abb. 2 Exemplarische Darstellung zweier rechts-bronchialer Doppellumentuben. Unterschiede gibt es vor allem hinsichtlich Cuff-Design, Länge und Winkel des bronchialen Tubusanteils, der farblichen Kodierung und des proximalen Endes (Bronchoskopie-Adapter, Verbindung zum Respirator).

mit integrierter Kamera, der eine Platzierung und eine kontinuierliche visuelle Überwachung gewährleisten kann ( Abb.4). Darüber hinaus gibt es auch spezielle Doppellumentrachealkanülen für Patienten mit einem Tracheostoma ( $\triangleright$ Abb. 4).

\section{Vor- und Nachteile}

Die Vorteile in der Anwendung eines DLT bestehen in

- der stabilen und zügigen Positionierung,

- der raschen Ent- und Belüftung der jeweiligen Lungenflügel und

- dem unproblematischen Wechsel von Ein- auf ZweiLungen-Ventilation oder von Ventilation der linken bzw. rechten Seite.

Die jeweiligen Lumen erlauben eine suffiziente Bronchialtoilette, die seitengetrennte Applikation eines positiven Atemwegsdruckes (CPAP) und eine fiberoptische Darstellung der Bronchien während thoraxchirurgischer Eingriffe. 


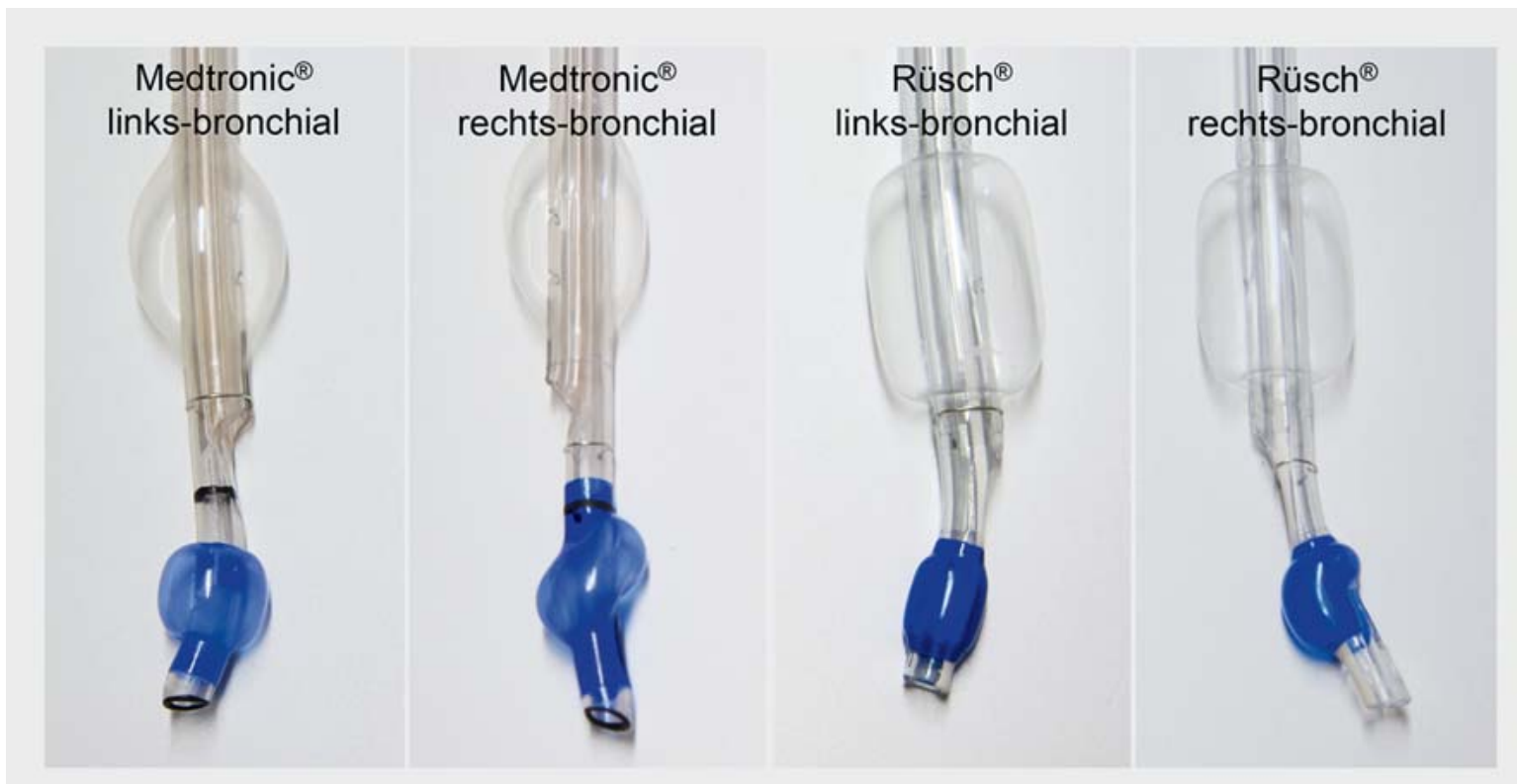

- Abb. 3 Exemplarische Darstellung der distalen Enden von linken und rechten Doppellumentuben. Unterschiede zeigen sich insbesondere im Cuff-Design, der Länge und der Winkel der bronchialen Tubusanteile.

- Tab. 1 Indikationen für eine Seitentrennung der Lungen.

\section{thoraxchirurgische Indikationen}

- Eingriffe am Lungenparenchym: Segmentresektion, Lobektomie, Pneumonektomie, Manschettenresektion, Lungentransplantation

- intrathorakale Eingriffe: Thorakoskopie, Ösophagusresektion, anteriore Wirbelsäuleneingriffe, thorakale Aortenaneurysmen, Thymektomie, Zwerchfelloperationen, Trichterbrustkorrekturen

\section{patientenbezogene Indikationen}

- Prävention der kontralateralen Lunge vor Infektion (Lungenabszess) oder Blut

- Verbesserung von Ventilation und Oxygenierung bei bronchopleuralen Fisteln, einseitigen Lungenerkrankungen (Bullae, Zysten)

- Tab. 2 Kenngrößen von Doppellumentuben [8].

\begin{tabular}{|l|l|l|l|}
\hline Tubus (Fr) jeweils links/rechts & Innendurchmesser ${ }^{\mathbf{1}} \mathbf{( m m )}$ & Außendurchmesser $\left.\mathbf{~}^{\mathbf{( m m}}\right)$ & Länge $\mathbf{( \mathbf { c m } )}$ \\
\hline $26 \mathrm{R}$ & $5,3 / 5,4$ & $9,8 / 9,3$ & $36,1 / 36,3$ \\
\hline $28 \mathrm{R}$ & $5,5 / 5,4$ & $10,2 / 10,1$ & $37,1 / 38,6$ \\
\hline $28 \mathrm{M}$ & 5 & 10,2 & 43,3 \\
\hline $32 \mathrm{M}$ & 5,4 & 11,2 & 43,3 \\
\hline $35 \mathrm{R}$ & $6,4 / 6,4$ & 12,5 & $39,5 / 39,9$ \\
\hline $35 \mathrm{M}$ & $7 / 6,9$ & $13 / 12,8$ & $43,8 / 43,2$ \\
\hline $37 \mathrm{R}$ & $6,8 / 6,9$ & $13,4 / 13,3$ & $40,4 / 41,3$ \\
\hline $37 \mathrm{M}$ & $7,6 / 7,5$ & $13,4 / 13,6$ & $43,5 / 43,2$ \\
\hline $39 \mathrm{R}$ & $7,3 / 7,4$ & 14,2 & $44,1 / 43$ \\
\hline $39 \mathrm{M}$ & $7,9 / 7,8$ & $14 / 14,5$ & $43,8 / 43,7$ \\
\hline $41 \mathrm{R}$ & $7,4 / 7,7$ & $14,3 / 14,6$ & $42,9 / 44,5$ \\
\hline $41 \mathrm{M}$ & $8 / 7,9$ & $14,4 / 14,6$ & $43,7 / 43,5$ \\
\hline
\end{tabular}

${ }^{1}$ gemessen am distalen Ende bronchial, ${ }^{2}$ mittig gemessen, Fr: French, R: Firma Rüsch, M: Firma Mallinckrodt 


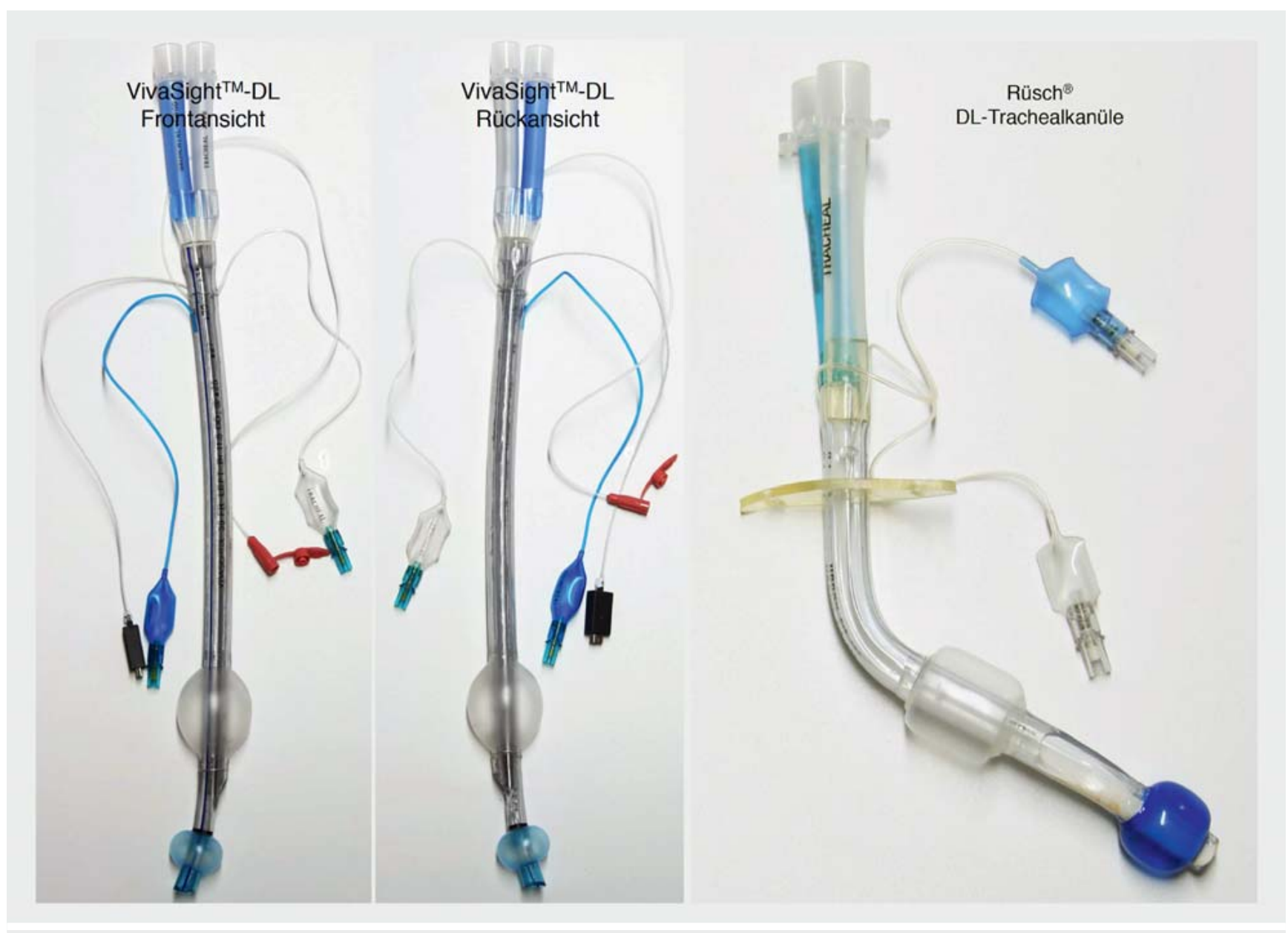

- Abb. 4 Exemplarische Darstellung eines VivaSight ${ }^{\mathrm{TM}}$-Doppellumentubus und einer Doppellumentrachealkanüle.

Die Nachteile liegen in

- dem größeren Atemwegstrauma bei der Intubation (mit Heiserkeit, Stimmbandschäden, trachealen oder bronchialen Rupturen),

- dem fixierten Design der Tuben (Größe, Länge, tracheobronchialer Winkel, Cuff-Volumen, > Abb. 1, 2 und 3),

- der Limitierung auf Erwachsene und Schulkinder und

- der Umintubation beim schwierigen Atemweg bzw. bei einer postoperativen Nachbeatmung.

Bei unsachgemäßer Anwendung von Doppellumentuben kann es zu Hypoxie, Hyperkapnie und Hyperinflation mit hämodynamischer Kompromittierung kommen [6, 7].

\section{Indikationen und Auswahl}

Die Indikationen für eine Seitentrennung der Lungen mit einem DLT lassen sich in den beiden Kategorien Thoraxchirurgie und Prävention von Kontamination zusammenfassen ( $\triangleright$ Tab. 1).
Bei der Auswahl sind 2 grundsätzliche Überlegungen zu berücksichtigen:

- Soll ein linker oder rechter Tubus zum Einsatz kommen?

- Welche Tubusgröße ist die richtige?

Um diese Fragen individualisiert zu beantworten, sind die Kenngrößen der einzelnen Doppellumentuben wichtig, die in $>$ Tab. 2 zusammengefasst sind.

\section{Linker oder rechter Tubus}

Die Asymmetrie in der tracheobronchialen Anatomie spiegelt sich im unterschiedlichen Design des linken und rechten DLT wider ( $\vee$ Abb. $\mathbf{3}$ ).

Wie bereits ausgeführt, ist der rechte Hauptbronchus im Vergleich zum linken deutlich kürzer: Der Abgang des rechten Oberlappenbronchus liegt sehr nahe der Hauptkarina und unterliegt auch reichlich anatomischen Variationen. Die Positionierung eines rechten DLT ist dadurch häufig schwieriger als die eines linken. Frühere Untersuchungen von Benumof et al. zur Sicherheitstoleranz 
\Tab. 3 Größenempfehlung für Doppellumentuben (DLT) (modifiziert nach [10, 11]).

\begin{tabular}{|c|c|c|c|c|c|}
\hline \multicolumn{2}{|l|}{ Frauen } & \multicolumn{2}{|l|}{ Männer } & \multirow{2}{*}{$\begin{array}{l}\text { gemessener trachealer } \\
\text { Durchmesser }(\mathrm{mm})\end{array}$} & \multirow[t]{2}{*}{ DLT (Fr) } \\
\hline Körpergröße (cm) & DLT (Fr) & Körpergröße (cm) & DLT (Fr) & & \\
\hline$<150$ & 32 & $<160$ & $35-37$ & 12,5 & 32 \\
\hline $150-160$ & 35 & $160-170$ & 37 & 14 & 35 \\
\hline$>160$ & $35-37$ & $>170$ & $37-39$ & 15 & 35 \\
\hline \multirow[t]{2}{*}{$>180$} & 39 & $>180$ & 41 & 16 & $37-39$ \\
\hline & & & & 18 & $39-41$ \\
\hline
\end{tabular}

beider Tuben kamen zu einer Spanne von 16-19 mm links und nur 1-4 mm rechts [9]. Die Anwendung des linken DLT garantiert bei thoraxchirurgischen Operationen bei korrekter Lage eine stabile Position, eine zuverlässige Seitentrennung und jeweils eine sichere Ventilation beider Lungenflügel.

\section{Merke}

Der linke DLT ist bevorzugt einzusetzen, insbesondere bei hilusfernen Resektionen und anderen intrathorakalen Eingriffen ohne Lungenparenchymverlust.

Die Dislokation des rechten Tubus durch intraoperative Manipulationen kann wiederum zu einer Verlegung des rechten Oberlappenbronchus führen und eine Hypoxie und Hyperkapnie während der Ein-Lungen-Ventilation begünstigen. Indikationen für den rechten Tubus sind unter „Info - Rechter DLT: Indikationen“ zusammengefasst.

\section{INFO}

Rechter DLT: Indikationen

Absolute und relative Indikationen für die Anwendung eines rechten DLT sind:

- linksseitige Pneumonektomie

- „Single-Lung“-Transplantation links

- endobronchiale Pathologie links (z. B. Tumorbefall, Kompression, Ruptur)

- Oberlappenmanschettenresektion links

Der Einsatz eines rechten DLT ist indiziert, wenn hilusnahe Präparation und Resektion unter Einbezug des linken Hauptbronchus geplant sind oder in der Erweiterung einer thoraxchirurgischen Operation auftreten könnten.

\section{Richtige Tubusgröße}

Kriterien für die individuelle Wahl der Tubusgröße können die Kombination von Geschlecht und Körpergröße sowie der Durchmesser der Trachea oder des linken Hauptbronchus in der CT sein [10-12]. Ein einziges Kriterium ist nicht ausreichend, und es existiert in den Fachkreisen kein Konsens für den besten Indikator. Die typischen DLT, die beim Erwachsenen zum Einsatz kommen, haben die Größen 35, 37, 39 und 41 French. Eine Entscheidung für die entsprechende Tubusgröße kann routinemäßig anhand der in > Tab. 3 dargestellten Kriterien erfolgen [10-12]. Die präoperative CT-Bildgebung ermöglicht dem Anästhesisten eine präzise Evaluation der individuellen tracheobronchialen Anatomie und der möglichen pathologischen Veränderungen, um den passenden DLT auszuwählen.

\section{Intubation mit einem DLT}

Der per se starre Doppellumentubus wird durch einen drahtförmigen Mandrin im bronchialen Lumen als Hilfsmittel bei der Intubation verstärkt. Die Beweglichkeit des Mandrins sollte insbesondere bei Biegung des Tubus durch den Einsatz eines wasserlöslichen Gleitgels erhöht werden. Ebenso sollte die Spitze des Mandrins proximal des bronchialen Tubusendes positioniert sein. Nach konventioneller direkter oder indirekter Laryngoskopie wird die nach anterior geneigte bronchiale Spitze des DLT durch die Glottisebene geführt. Nach Zurückziehen des Mandrins wird dann der DLT in einer $90^{\circ}$-Rotationsbewegung je nach Tubus zur linken oder rechten Seite vorgeschoben. Ein kleiner Widerstand ist sowohl beim Durchtritt der Querschnittsvergrößerung des trachealen Endes des Tubus durch die Stimmbandebene zu spüren als auch beim Erreichen der Hauptkarina. Die Platzierung in den jeweiligen Hauptbronchus sollte jedoch ohne zu großen Widerstand erfolgen. Bei weiteren Widerständen oder auch sehr kleinen Patienten (Cave: vergleichbare Länge aller Tuben!) ist eine fiberoptische Positionierung durch das bronchiale Lumen zwingend erforderlich.

\section{Merke}

Nach Blocken des trachealen Cuffs, Anschluss des Y-Konnektors und Etablieren der mechanischen Beatmung beider Lungen muss eine fiberoptische Lagekontrolle des Doppellumentubus erfolgen. 


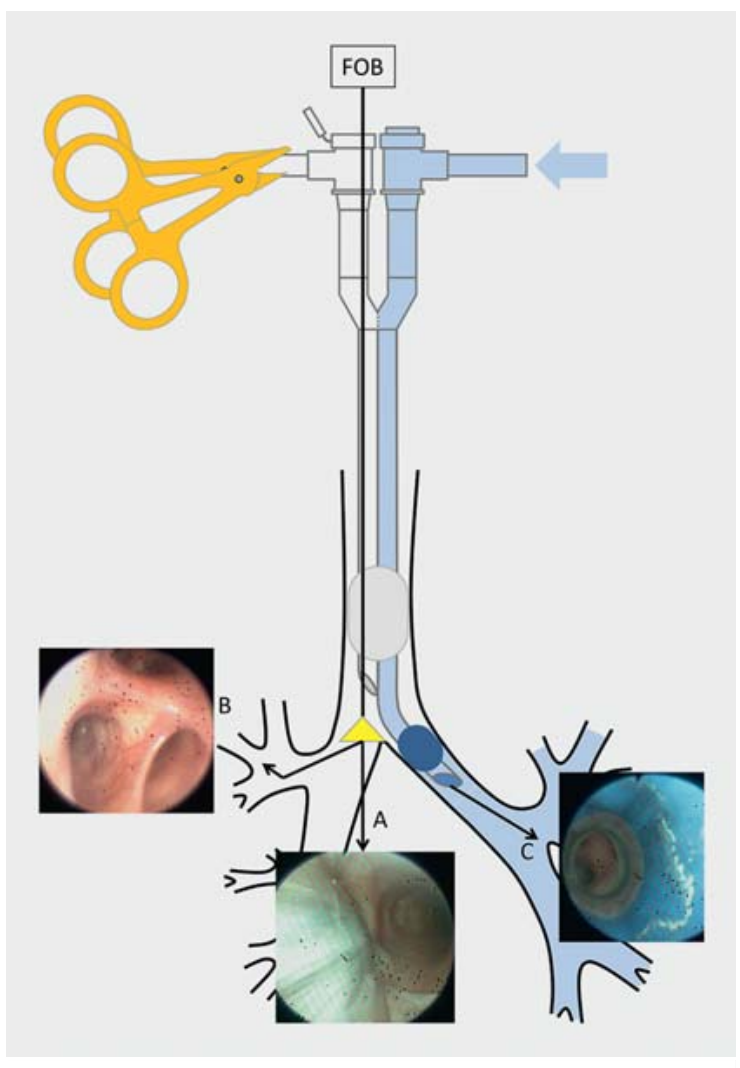

- Abb. 5 Fiberoptisch bronchoskopische (FOB) Evaluation der korrekten Lage eines Doppellumentubus links. A: Blick durch das tracheale Lumen des Doppellumentubus auf die Karina und den rechten Hauptbronchus mit links-bronchialer Intubation. B: Blick durch das tracheale Lumen auf die Trisegmentstruktur des rechten Oberlappens. C: Blick durch das bronchiale Lumen des Doppellumentubus auf die Ober-/Unterlappenkarina links-bronchial.

\section{Lagekontrolle}

Die korrekte Lage des DLT ist für die sichere Seitentrennung der Lungen unabdingbar und heutzutage ausschließlich über eine fiberoptische Bronchoskopie (FOB) durch beide Lumina zu gewährleisten. Eine alleinige klinische, visuelle oder auskultatorische Evaluation als Lagekontrolle ist nicht ausreichend.

Die FOB hat zunächst durch das tracheale Lumen mit der Darstellung der Karina die korrekte Intubation des jeweiligen Hauptbronchus zu prüfen. Ebenso ist zu evaluieren, ob alle Ostien der Lappenbronchien zugänglich sind. Die Lage ist korrekt, wenn der endobronchiale Cuff direkt an der Karina liegt und nicht herniert. Beim Einsatz des linken DLT kann so der Abgang des rechten Oberlappenbronchus verifiziert werden ( $\bullet$ Abb. 5 und Video 1). Beim rechten DLT kommen die Karina und der linke Hauptbronchus mit rechts-bronchialer Intubation zur Darstellung ( $\triangleright$ Abb. 6 und Video 2 ).

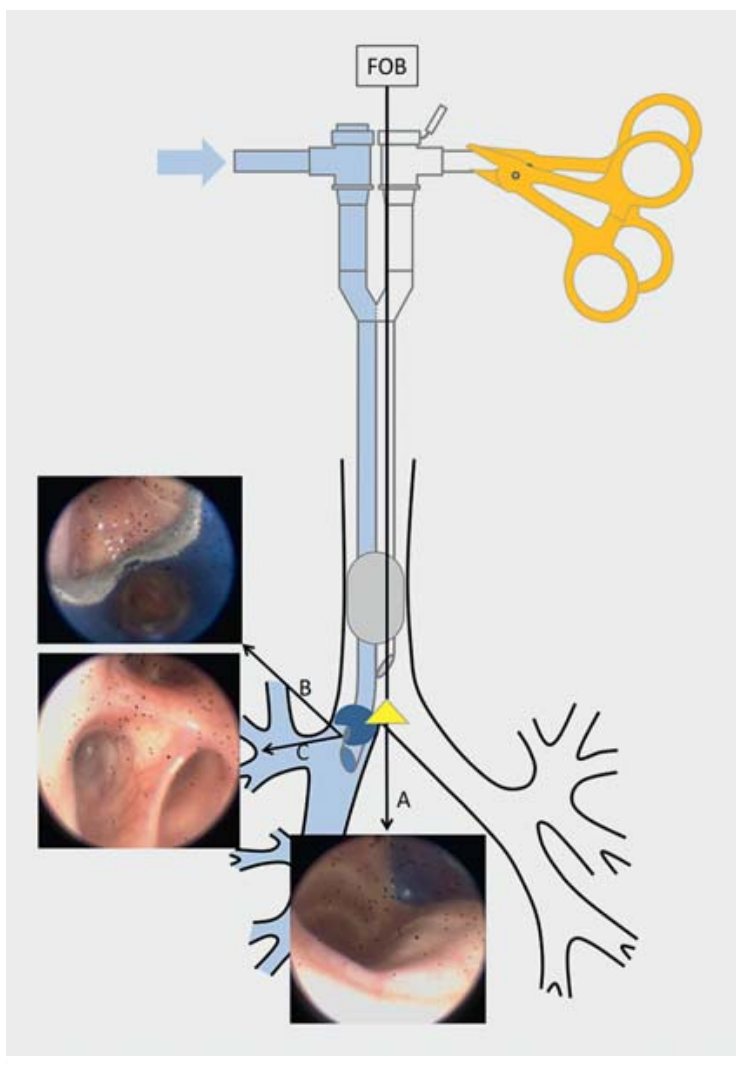

Abb. 6 Fiberoptisch bronchoskopische (FOB) Evaluation der korrekten Lage eines Doppellumentubus rechts. A: Blick durch das tracheale Lumen des Doppellumentubus auf die Karina und den linken Hauptbronchus mit rechts-bronchialer Intubation. B und C: Blick durch das bronchiale Lumen und das Murphy-Auge auf die Trisegmentstruktur des rechten Oberlappens.

VIDEO
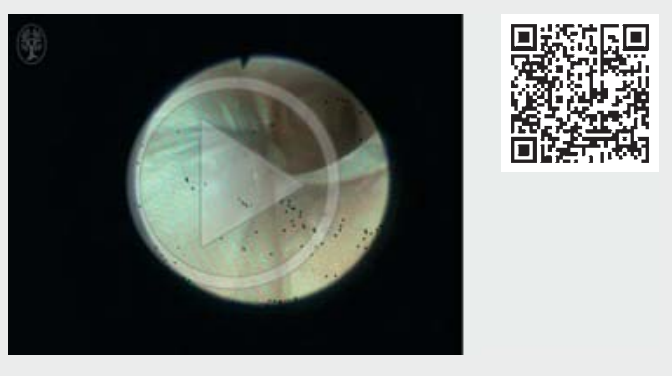

- Video 1 Links-bronchialer Doppellumentubus, fiberoptische Bronchoskopie des trachealen Lumens: Karina und rechter Hauptbronchus mit links-bronchialer Intubation sowie Blick auf die Trisegmentstruktur des rechten Oberlappens. 

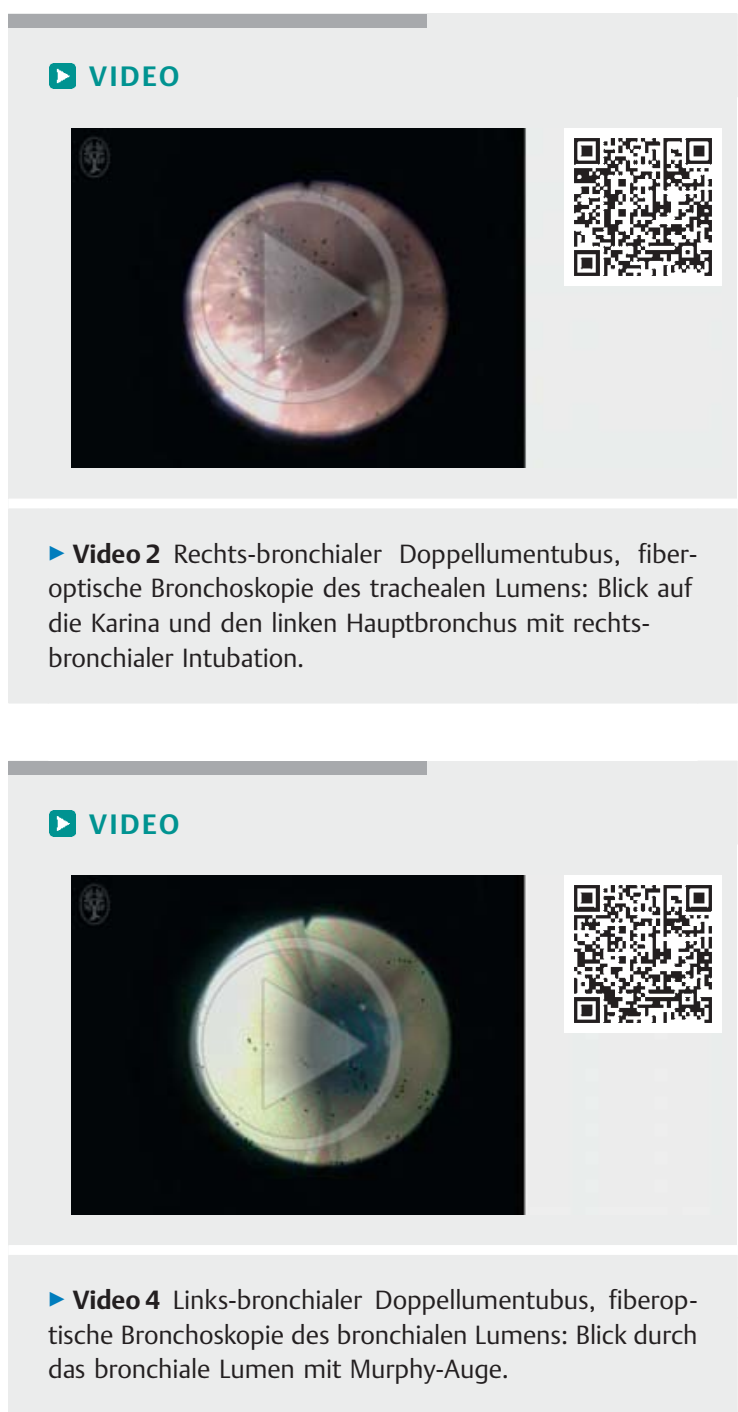

Im Anschluss muss mittels FOB durch das bronchiale Lumen der Abstand zur Ober-/Unterlappenkarina links (linker DLT) dargestellt werden ( $\bullet$ Abb. 5, Video 3 und 4 ). Bei einem rechten DLT muss die FOB durch das bronchiale Lumen die präzise Position des Doppellumentubus vor dem rechten Oberlappen zeigen ( $\triangleright$ Abb. 6 und Video 5).

Der endobronchiale Cuff sollte nach endgültiger Lagerung unter Sicht mit einem minimalen Volumen hauptkarinanah geblockt werden.

\section{Cave}

Da thoraxchirurgische Eingriffe überwiegend in Seitenlage durchgeführt werden, ist eine Tubusdislokation bei jeder Bewegung des Patienten möglich und eine Reevaluation indiziert.

\section{Komplikationen und Fehllagen}

Neben den bekannten Komplikationen endotrachealer Intubationen (Zahnschäden u. a.) kommt es beim Einsatz

\section{VIDEO}

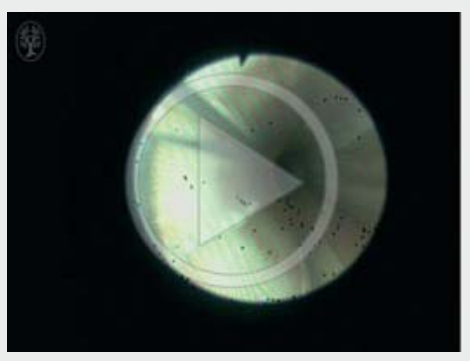

- Video 3 Links-bronchialer Doppellumentubus, fiberoptische Bronchoskopie des bronchialen Lumens: Blick auf die Ober-/Unterlappenkarina links-bronchial.

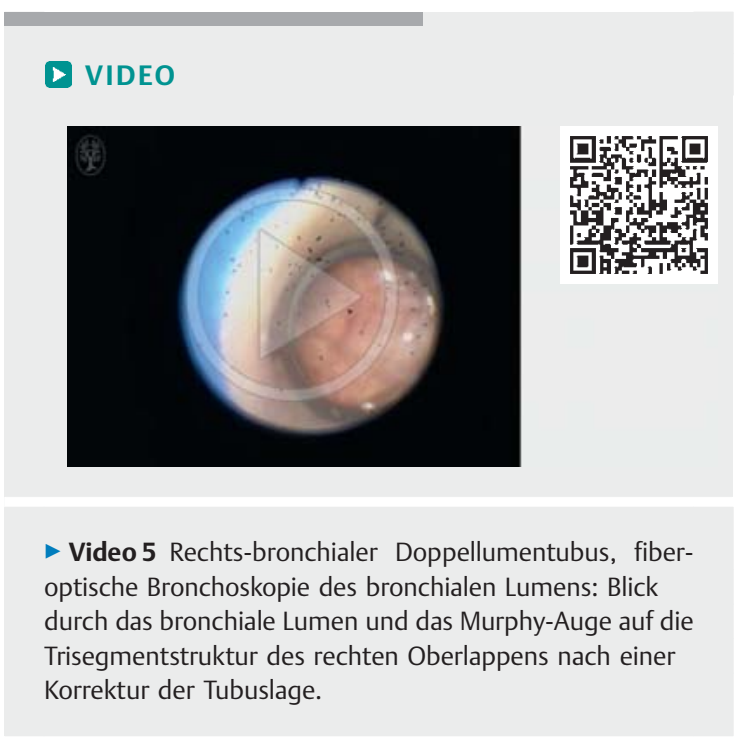

von Doppellumentuben häufiger zu Verletzungen der Trachea und der Hauptbronchien. Neben dem Durchmesser und der Steifigkeit führen Fehllagen, eine zu distale Platzierung, Rotationsbewegungen beim Einführen, Verletzungen durch den Mandrin und Überblockungen des Cuffs zu Komplikationen. Um diese zu vermeiden, hat die Intubation achtsam zu erfolgen. Der Mandrin ist bei Durchtritt durch die Glottisebene zu entfernen.

\footnotetext{
Merke

Ein Widerstand beim Vorschieben sollte erkannt und visualisiert werden, da dieser sowohl das Erreichen der Hauptkarina als auch eine zu tiefe Intubation anzeigt.
}

Alternativ zu dem oben beschriebenen Vorgehen könnte - direkt nach Entfernung des Mandrins - der Tubus durch eine FOB-geführte (über das bronchiale Lumen) Intubation platziert werden. 

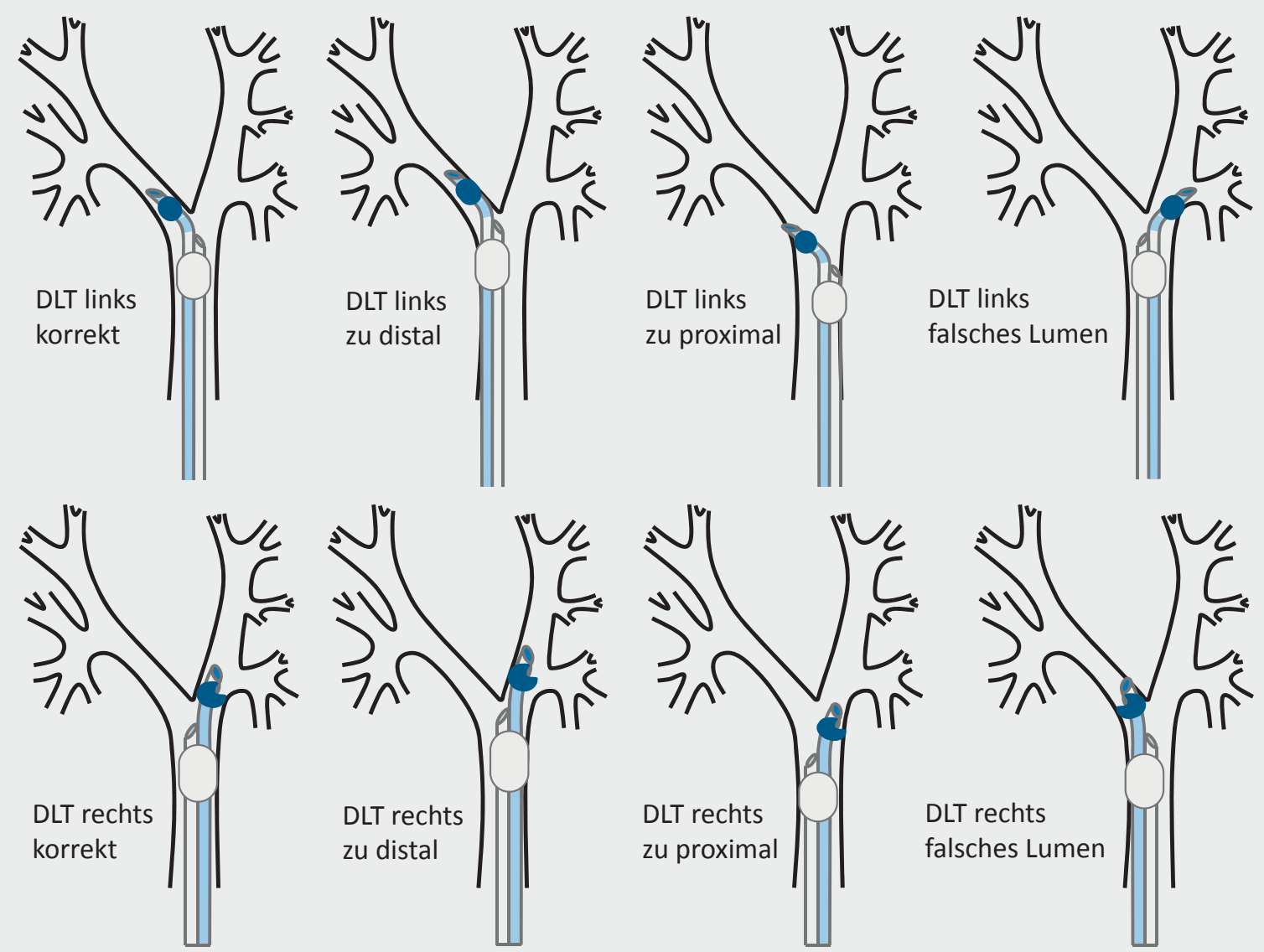

- Abb. 7 Übersicht der endotrachealen und -bronchialen Fehllagen von Doppellumentuben (DLT).

\section{INFO}

Fehllagen - Übersicht

Die verschiedenen Fehllagen von Doppellumentuben können unter folgender Systematik zusammenge-

fasst werden ( $\triangleright$ Abb. 7) [6]:

- zu tief oder zu distal

- zu proximal

- falsches Lumen

\section{Zu distal, zu proximal}

Bei der zu tiefen (distalen) Position des linken DLT erkennt man durch die FOB tracheal die Mukosa des linken Hauptbronchus. Durch die Tubuswand sieht man als Schatten das Lumen des rechten Hauptbronchus. Bei der bronchialen FOB erkennt man die Ober-/Unterlappenkarina links oder nur bronchiale Strukturen. Die Korrektur erfolgt durch das Zurückziehen des DLT bei gleichzeitiger FOB tracheal, bis die Hauptkarina sichtbar wird [6].

Bei der zu proximalen Position des linken DLT erkennt man durch die FOB tracheal die Mukosa der Trachea und keine Schatten des rechten Hauptbronchus. Möglicher- weise werden der bronchiale Anteil mit blauem Cuff oder auch die Hauptkarina bei der weiteren FOB sichtbar. Die bronchiale FOB zeigt entweder die Hauptkarina oder Strukturen des linken bzw. rechten Bronchialsystems. Die Korrektur erfolgt während trachealer FOB durch Vorschieben des Tubus, bis die Hauptkarina sichtbar wird und der Hauptbronchus intubiert werden kann [6].

\section{Falsches Lumen}

Bei der endobronchialen Intubation des rechten Hauptbronchus mit einem linken DLT erkennt man über die tracheale FOB nur Mukosa und kein weiteres Lumen (z. B. rechter Hauptbronchus). Die bronchiale FOB zeigt die Anatomie des Bronchus intermedius. Bei der Korrektur ergeben sich 2 Optionen:

- FOB bronchial, Zurückziehen des Tubus, bis sich die Hauptkarina zeigt, Intubation des linken Hauptbronchus und Lagekontrolle über die tracheale FOB.

- FOB tracheal, Zurückziehen des Tubus von etwa $5 \mathrm{~cm}$, Rotation des Tubus gegen den Uhrzeigersinn und Vorschieben, bis die Hauptkarina sichtbar wird. Lagekontrolle über eine bronchiale FOB. 
Bei der endobronchialen Intubation des linken Hauptbronchus mit einem rechten DLT erkennt man über die tracheale FOB die Anatomie des rechten Hauptbronchus. Die bronchiale FOB zeigt die Anatomie der linken Ober-/ Unterlappenkarina. Die Korrektur erfolgt in vergleichbarer Art und Weise wie oben formuliert.

\section{Vorgehen bei Platzierungsproblemen}

Bei einer primären Unsicherheit, wo der platzierte DLT liegt, ist eine bronchiale FOB hilfreich. Beim Zurückziehen von Fiberoptik und Tubus wird die Hauptkarina sichtbar werden.

\section{Merke}

Für die Schwierigkeit, den DLT durch die Glottisebene vorzuschieben, ergeben sich mehrere Gründe: Der DLT ist zu groß, blockiert an einer Trachealspange, oder es handelt sich um einen schwierigen Atemweg.

Bei Letzterem sollte neben dem Algorithmus „schwieriger Atemweg“ auf eine Larynxmaske oder einen Einlumentubus gewechselt werden und über eine FOB die Ursache verifiziert werden. Falls kein Befund erhoben werden kann, kann über einen „Airway-Exchange“-Katheter auf einen möglicherweise kleineren DLT gewechselt werden.

Bei Problemen, den linken DLT korrekt zu platzieren, könnten neben der Tubusgröße anatomische Besonderheiten der Hauptkarina ursächlich sein. Neben der Intubation mit einem kleineren Tubus unter FOB könnte ein Wechsel auf einen rechten Tubus oder ein Bronchusblockersystem hilfreich sein.

Beim Einsatz eines rechten Tubus kommt es mitunter zu Problemen, das bronchiale Fenster exakt über dem Ostium des rechten Oberlappens zu positionieren. Zunächst ist es wichtig, während der FOB den Port über Zurückziehen oder Vorschieben zu platzieren oder über eine Rotation (meist im Uhrzeigersinn) das Ostium aufzusuchen. Es empfiehlt sich, über die tracheale FOB die bronchiale Cuff-Position zu evaluieren und den Cuff schon in Rückenlage zu blockieren. So kann die Position für die Lagerungsmaßnahmen schon etwas fixiert werden. Durch die Körpergröße oder anatomische Besonderheiten kann es zur Herniation des bronchialen Cuffs über die Hauptkarina mit entsprechender Leckage kommen. Wenn dann keine suffiziente Seitentrennung oder Ventilation der rechten Lunge möglich ist, sollte auf ein Bronchusblockersystem mit Einlumentubus gewechselt werden.

\section{Ausbildung}

Formal existieren keine Ausbildungsrichtlinien für Anästhesisten, um sich die Kenntnisse der bronchoskopischen Anatomie anzueignen. Eine 1:1-Schulung, Videos und Simulationen sowie die Teilnahme an Bronchoskopiesemi- naren sind hilfreich $[13,14]$. Weitere interessante Links und Apps sind:

- http://www.thoracic-anesthesia.com

- http://www.bronchoscopy.nl

- http://www.orsim.co.nz/home: kleiner Bronchoskopiesimulator

- Fibreoptic Scope Size: App für die Größenkompatibilität von Atemwegshilfen und Fiberoptik

- Double Lumen (Crystal Clear Solutions Version 2.2): App mit 18 Simulationen verschiedener Lagekontrollen

- Broncho (Bronchoscopy Foundation): App über tracheobronchiale Anatomie

\section{KERNAUSSAGEN}

- Die Seitentrennung der Lungen mit konsekutiver Ein-Lungen-Ventilation ist der zentrale Bestandteil des Atemwegsmanagements in der Thoraxanästhesie und mit dem Doppellumentubus effektiv und sicher zu erreichen.

- Neben thoraxchirurgischen und prozedurbezogenen Indikationen umfasst das Indikationsspektrum für die Seitentrennung der Lungen auch patientenspezifische Faktoren (z. B. Prävention von Kontamination).

- Die Kenntnis der tracheobronchialen Anatomie, der standardmäßige Einsatz einer flexiblen Fiberoptik und die Expertise des verantwortlichen Anästhesisten sind Grundvoraussetzungen für das Management mit dem Doppellumentubus.

- Aufgrund der anatomischen Gegebenheiten ist die Positionierung eines rechts-bronchialen Doppellumentubus häufig schwieriger als die eines linksbronchialen. Der linke DLT ist daher bevorzugt einzusetzen, insbesondere bei hilusfernen Resektionen und anderen intrathorakalen Eingriffen ohne Lungenparenchymverlust.

- Nach der Platzierung des Doppellumentubus muss eine Lagekontrolle mittels fiberoptischer Bronchoskopie durch beide Lumina erfolgen.

- Eine erneute fiberoptisch bronchoskopische Evaluation der korrekten Lage des Doppellumentubus ist nach jeder Umlagerung des Patienten während der Operation erforderlich.

- Zum Erlernen des praktischen Managements im klinischen Alltag sind 1: 1-Schulung, Videos, Simulationen und die Teilnahme an Bronchoskopieseminaren hilfreich.

\section{Interessenkonflikt}

Die Autoren erklären, dass keine Interessenkonflikte vorliegen. 


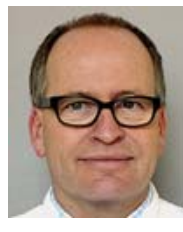

\section{Torsten Loop}

Prof. Dr. med., Jahrgang 1960. 1983-1989 Studium der Humanmedizin in Freiburg, 1991 Promotion. 1991-1992 Assistenzarzt Kantonsspital Liestal/Schweiz. 1992-1995 Wissenschaftlicher Assistent an der Anästhesiologischen Universitätsklinik Freiburg. Seit 1996 Facharzt in allen chirurgisch operativen Bereichen, Anästhesiologische Universitätsklinik Freiburg. 2006 Habilitation. 2010 Außerplanmäßige Professur. Seit 2011 Leitender Oberarzt.

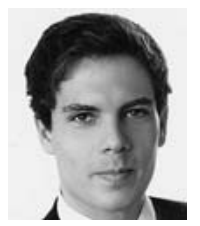

\section{Johannes Spaeth}

Dr. med., Jahrgang 1980. 2003-2010 Studium der Humanmedizin. 2015 Promotion. Seit 02/ 2011 Arzt in Weiterbildung am Universitätsklinikum Freiburg, Klinik für Anästhesie und Intensivmedizin.

Korrespondenzadresse

Prof. Dr. med. Torsten Loop

Klinik für Anästhesiologie und Intensivmedizin

Universitätsklinikum Freiburg

Hugstetter Straße 55

79106 Freiburg

torsten.loop@uniklinik-freiburg.de

\section{Wissenschaftlich verantwortlich} gemäß Zertifizierungsbestimmungen

Wissenschaftlich verantwortlich gemäß Zertifizierungsbestimmungen für diesen Beitrag ist Prof. Dr. med. Torsten Loop, Freiburg.

\section{Literatur}

[1] Falzon D, Alston RP, Coley E et al. Lung isolation for thoracic surgery: from inception to evidence-based. J Cardiothorac Vasc Anesth 2017; 31: 678-693

[2] Conacher ID. Implications of a tracheal bronchus for adult anaesthetic practice. $\mathrm{Br}$ ] Anaesth 2000; 85: 317-320
[3] Read R, St Cyr J, Marek J et al. Bronchial anomaly of the right upper lobe. Ann Thorac Surg 1990; 50: 980-981

[4] Beder S, Kupeli E, Karnak D et al. Tracheobronchial variations in Turkish population. Clin Anat 2008; 21: 531-538

[5] Robertshaw FL. Low resistance double-lumen endobronchial tubes. Br ] Anaesth 1962; 34: 576-579

[6] Karzai W, Schwarzkopf K. Hypoxemia during one-lung ventilation: prediction, prevention, and treatment. Anesthesiology 2009; 110: 1402-1411

[7] Spaeth J, Ott M, Karzai W et al. Double-lumen tubes and autoPEEP during one-lung ventilation. $\mathrm{Br}$ J Anaesth 2016; 116: $122-130$

[8] Spaeth J, Hojnik A, Ott M et al. Characteristics of double-lumen tubes determine bronchial airway pressure. J Cardiothorac Vasc Anesth 2016; 30: 954-960

[9] Benumof JL, Partridge BL, Salvatierra C et al. Margin of safety in positioning modern double-lumen endotracheal tubes. Anesthesiology 1987; 67: 729-738

[10] Slinger P. A view of and through double-lumen tubes. J Cardiothorac Vasc Anesth 2003; 17: 287-288

[11] Brodsky JB, Macario A, Mark JB. Tracheal diameter predicts double-lumen tube size: a method for selecting left double-lumen tubes. Anesth Analg 1996; 82: 861-864

[12] Brodsky JB, Lemmens HJ. Tracheal width and left double-lumen tube size: a formula to estimate left-bronchial width. J Clin Anesth 2005; 17: 267-270

[13] Wong AK. Full scale computer simulators in anesthesia training and evaluation. Can J Anaesth 2004; 51: 455-464

[14] Haponik EF, Russell GB, Beamis JF jr. et al. Bronchoscopy training: current fellows' experiences and some concerns for the future. Chest 2000; 118: 625-630

\section{Bibliografie}

DOI https://doi.org/10.1055/s-0043-114678 Anästhesiol Intensivmed Notfallmed Schmerzther 2018; 53: 174-185 @ Georg Thieme Verlag KG Stuttgart · New York ISSN 0939-2661 


\section{Punkte sammeln auf CME.thieme.de}

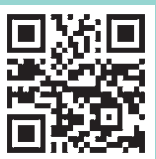

Diese Fortbildungseinheit ist 12 Monate online für die Teilnahme verfügbar.

Sollten Sie Fragen zur Online-Teilnahme haben, finden Sie unter cme.thieme.de/hilfe eine ausführliche Anleitung. Wir wünschen viel Erfolg beim Beantworten der Fragen!

Unter eref.thieme.de/ZZX8XER oder über den QR-Code kommen Sie direkt zum Artikel zur Eingabe der Antworten.

VNR 2760512018154654784

\section{Frage 1}

Welche Aussage zum Doppellumentubus (DLT) ist richtig?

A Moderne DLT können sowohl für eine linke als auch rechte endobronchiale Intubation verwendet werden.

B Moderne DLT unterscheiden sich im Design des linken und des rechten DLT.

C Moderne DLT besitzen keinen Mandrin.

D Das bronchiale Lumen ist farblich nicht gekennzeichnet.

E Rechte DLT werden häufiger eingesetzt als linke DLT.

\section{Frage 2}

Welche Aussage zum linken DLT ist richtig?

A Der linke DLT besitzt eine größere Sicherheitstoleranz als der rechte DLT.

B Der linke DLT ist schwieriger endobronchial zu platzieren.

C Der linke DLT disloziert leichter.

D Der linke DLT induziert keine Atemwegstraumata.

E Der linke DLT besitzt distal nur ein kleines Lumen.

\section{Frage 3}

Welche Aussage ist richtig? Die Lagekontrolle eines DLT soll...

A ausschließlich klinisch erfolgen.

$B$ eine Fehllage mittels Auskultation ausschließen.

C grundsätzlich fiberoptisch erfolgen.

D nur nach Intubation erfolgen.

E der Thoraxchirurg vornehmen.

\section{Frage 4}

Welcher der nachfolgend genannten ist der wahrscheinlich adäquate Tubus für eine Frau mit der Körpergröße $160 \mathrm{~cm}$ und einer geplanten Pleurodese links?
A DLT $37 \mathrm{Fr}$ rechts
B DLT $39 \mathrm{Fr}$ links
C DLT 35 Fr rechts
D DLT 35 Fr links
E Einlumentubus 7,0

\section{Frage 5}

Welcher der nachfolgend genannten ist der wahrscheinlich adäquate Tubus für einen Mann mit der Körpergröße $182 \mathrm{~cm}$ zur Ösophagusresektion?
A DLT 28 Fr links
B DLT $41 \mathrm{Fr}$ links
C DLT $39 \mathrm{Fr}$ rechts
D Einlumentubus plus Bronchusblocker
E DLT $41 \mathrm{Fr}$ rechts

\section{Frage 6}

Welcher der nachfolgend genannten ist der wahrscheinlich adäquate Tubus für eine linksseitige Pneumonektomie bei einem großen zentralen Tumor bei einem 53-jährigen Patienten?
A DLT rechts
B DLT links
C Einlumentubus plus Bronchusblocker
D endobronchiale Intubation mit einem Einlumentubus rechts
E Einlumentubus und Einsatz einer ECMO

\section{Frage 7}

Welcher der folgenden Eingriffe stellt keine Indikation für eine Seitentrennung der Lungen dar?
A Zwerchfelloperation
B Manschettenresektion
C anteriorer Wirbelsäuleneingriff
D Thyreoidektomie
E Thymektomie

\section{- Weitere Fragen auf der folgenden Seite...}




\section{Punkte sammeln auf CME.thieme.de}

\section{Fortsetzung $\ldots$}

\section{Frage 8}

Welche Aussage ist richtig? Der linksseitige DLT ...

A liegt stabiler als der rechte DLT.

B liegt im rechten Hauptbronchus, wenn bei der Bronchoskopie über den trachealen Schenkel der linke Hauptbronchus sichtbar ist.

C liegt zu tief, wenn die Hauptkarina bei der Bronchoskopie über den trachealen Schenkel sichtbar ist.

D liegt zu proximal, wenn bei der Bronchoskopie über den trachealen Schenkel der rechte Oberlappen verlegt ist.

E liegt korrekt, wenn bei der Bronchoskopie über den bronchialen Schenkel der rechte Hauptbronchus durch den Tubus sichtbar ist.

\section{Frage 9}

Welche Aussage ist richtig? Der rechtsläufige DLT ...

$A$ ist gefährlich.

B liegt stabiler als der linke DLT.

C besitzt zusätzlich einen Port zur Ventilation des rechten Unterlappens.

D besitzt ein spezielles Cuff-Design.

E kann auch endobronchial links verwendet werden.

\section{Frage 10}

Welche Aussage ist falsch?

A Bei einem Patienten zur geplanten Pleurektomie und atypischen Resektion bei rezidivierenden Pneumothoraces ist ein DLT indiziert.

B Bei einem Patienten zur Lungentransplantation ist aufgrund des Einsatzes einer Herz-Lungen-Maschine kein DLT zur Seitentrennung der Atemwege notwendig.

C Wenn postoperativ aufgrund einer intraoperativen respiratorischen Insuffizienz die Indikation zur Nachbeatmung gestellt wird, sollte der DLT nicht belassen werden.

D Die jeweiligen Lumen des DLT erlauben die seitengetrennte Applikation eines positiven Atemwegsdruckes (CPAP).

E Der Einsatz eines DLT ist auf Erwachsene und Schulkinder limitiert. 